\title{
Saccharomyces Cerevisiae Immobilized in Alginate for Continuous Fermentation
}

\author{
Zhaohui Xing, Qi Zhang, Xueyi Shi, and Yan Lin
}

\begin{abstract}
Saccharomyces cerevisiae BY4742 cells were immobilized in alginate and applied for continuous fermentation. Immobilization of yeast cell showed technical and economic advantages over free cell system. In the fermentation, the initial glucose concentration was $110 \mathrm{~g} / \mathrm{L}$. The hydraulic retention time (HRT) of fermentation broth was set to $24 \mathrm{~h}$ with a re-activate time of $12 \mathrm{~h}$ after first incubation period $72 \mathrm{~h}$. Ethanol yield and glucose utilizing ratio were analyzed for verifying the performance of continuous fermentation and the improvement of immobilized yeasts over free yeast cells for fermentation. Comparing dates in two experimental groups, the ethanol yield could reach to about $80 \%$ and the glucose utilizing ratio had the similar pattern with averagely $50 \%$. The ethanol yield and glucose utilizing ratio had a significant improvement with the pre-activation and the re-activation.
\end{abstract}

Index Terms-Saccharomyces cerevisiae, yeast immobilization, continuous fermentation, fermentation efficiency.

\section{INTRODUCTION}

Because of global energy crisis with diminishing fossil fuels and environmental problems such as global warming and air pollution, growing attention has been devoted in the past few years to the ethanol production from renewable carbohydrate materials which is widely recognized as an alternative to fossil fuels with powerful economic, environmental and strategic benefits, and much research has focused on ethanol production using immobilized viable microbial cells in continuous systems [1], [2]. Traditional fermentation systems use freely suspended yeast cells in a batch bioreactor. The whole reactor volume is gradually fermented and subsequently removed from the reactor in which yeast cells can be adversely influenced by other parameters and inhibition can be caused either by product or substrate concentration [3]. During batch fermentation of free cells, product ethanol came out of the system with fermentation broth containing free cells, glucose and others impurities; it is hard to separate mixture components for ethanol only [4]. Recently the immobilized biocatalysts have been extensively investigated. Immobilized cells show many advantages over free cell in tolerance of temperature and $\mathrm{pH}$, and can provide high cell activity in the reactors.

Manuscript received August 26, 2014; revised January 26, 2015. This work was supported in part by The Major Science and Technology Program sponsored the research project for Water Pollution Control and Treatment (2009ZX07101-015-003) and the Shanghai Natural Science Foundation (No. 11ZR1417200).

Zhaohui Xing, Yan Lin, and Qi Zhang are with the Shanghai Jiao Tong University, Shanghai 200240, China (e-mail: doulejuice2013@sjtu.edu.cn, linyansjtu@126.com, 249716843@qq.com). Xueyi
China.
There are several operational problems effecting continuous usability of continuous fermentation system. Continuous fermentation systems with immobilized yeast cells have a continuous flow of fermented medium into the fermenter and a corresponding continuous flow of fermented product out of the system, which means the nutrient utilization in, the single stage continuous stirred tank reactor (CSTR) for example, is lower than batch fermentation. Besides the designs of reactor system, such as the pre-activation time, the hydraulic retention time (HRT) and the reactor volume, have an impact on the experimental results [5].

The aim of this study was to obtain high ethanol production by immobilizing yeast cells in alginate, and apply these immobilized beads for continuous fermentation to test the fermentation performance of immobilized yeast [6], improve the designs of continuous fermentation according the experimental results, and offer reference for following development works.

\section{MATERIAL AND METHODS}

\section{A. Yeast Strain and Culture Condition}

S. cerevisiae BY4742 (originally from EURSCAF, Germany) used in this study was thermo tolerant strain selected and maintained in glycerol vials at $-80^{\circ} \mathrm{C}$ for use as a working stock in our laboratory (School of Environmental Science and Engineering, Shanghai Jiao Tong University, China). Active cultures for inoculation were obtained in $1 \mathrm{~L}$ Erlenmeyer flasks with $500 \mathrm{~mL}$ of growth medium containing $20 \mathrm{~g} / \mathrm{L}$ glucose, $20 \mathrm{~g} / \mathrm{L}$ peptone, and $10 \mathrm{~g} / \mathrm{L}$ yeast extract. The liquid medium was sterilized at $121{ }^{\circ} \mathrm{C}$ for 30 $\min$. The pre-culture was performed in a rotatory shaker at $37^{\circ} \mathrm{C}$ and $180 \mathrm{rpm}$ for $16 \mathrm{~h}$ and then used to inoculate 500 $\mathrm{mL}$ baffled shake-flasks containing $250 \mathrm{~mL}$ of the above medium with the same condition. The cells were centrifuged at $3000 \mathrm{rpm}$ for $3 \mathrm{~min}$, the supernatant was decanted, and the cells were washed three times with sterile water [7].

\section{B. Preparation for the Carriers}

The obtained cells were re-suspended in sterile water and used as inoculum in the continuous fermentation. The cells count was determined at $\mathrm{OD}_{600}$. Sodium alginate gel was obtained in $100 \mathrm{~mL}$ beaker with $45 \mathrm{~mL}$ sterile water containing $1.875 \mathrm{~g}$ sodium alginate. The medium was sterilized at $121{ }^{\circ} \mathrm{C}$ for $30 \mathrm{~min}$.

\section{Immobilization of Yeast}

The immobilization in calcium alginate beads was carried out as followed: The yeast cells slurry (containing yeast $1.5 \mathrm{~g}$ ) and the sodium alginates were mixed with sterile water to 
final volume $300 \mathrm{~mL}$, with $2.5 \%$ (w/v) sodium alginate and $2 \%$ $(\mathrm{w} / \mathrm{v})$ yeast cells slurry. The mixture obtained was extruded dropwise with sterile syringes into gently stirred $2 \%(\mathrm{w} / \mathrm{v})$ $\mathrm{CaCl}_{2}$ and kept for $4 \mathrm{~h}$ for stabilization. The beads obtained containing cells were stored at $4{ }^{\circ} \mathrm{C}$ and used for further studies.

\section{Continuous Fermentation}

Two control groups were used for the study. The beads in group 1 (G1) were direct used for fermentation. The beads in group 2 (G2) were activated before fermentation with active cultures for inoculation above. There were 2 reactors (1\# and $2 \#)$ in each experiment group. Reaction conditions were as follows: the initial glucose concentrations were $110 \mathrm{~g} / \mathrm{L}$, with the cell concentration $4.6 \mathrm{~g} / \mathrm{L}$. The reactors were performed at a temperature of $35^{\circ} \mathrm{C}$ and an initial $\mathrm{pH}$ of 4.0. Stirring speed was set as $180 \mathrm{rpm}$, and the incubation period was $144 \mathrm{~h}$. Samples were token per $12 \mathrm{~h}$ or $24 \mathrm{~h}$. Two reactors contained fermentation broth $200 \mathrm{~mL}$ with $60 \mathrm{~mL}$ immobilized beads. The HRT of fermentation broth was set to $24 \mathrm{~h}$ with a re-activate time of $12 \mathrm{~h}$ after first incubation period $72 \mathrm{~h}$.

\section{E. Analytical Method}

Ethanol and reduce sugar samples were all diluted 10 times and analyzed by high performance liquid chromatography (HPLC LC-10AD, SHIMADZU, Kyoto, Japan) equipped with a refractive index detector (RID-10A, SHIMADZU, Kyoto, Japan). An Aminex HPX-87H column (Bio-Rad, USA) with a safe guard column operated at $65^{\circ} \mathrm{C}$ using pure grade water as the mobile phase $(0.8 \mathrm{~mL} / \mathrm{min})$ was used for the separation. In nutrient for continuous fermentation, only glucose can be fermented into ethanol by $S$. cerevisiae BY4742, so the maximum theoretical ethanol from glucose could be calculated according to the stoichiometric relationship represented by (1), i.e. $100 \mathrm{~g}$ of glucose can produce $51.1 \mathrm{~g}$ of ethanol. The ethanol yield was calculated according to (2). The glucose utilizing ratio is calculated according to (3).

$$
\begin{gathered}
\mathrm{C}_{6} \mathrm{H}_{12} \mathrm{O}_{6} \rightarrow 2 \mathrm{CH}_{3} \mathrm{CH}_{2} \mathrm{OH}+2 \mathrm{CO}_{2} \\
Y_{E}=\frac{C_{E t h}}{C_{G 0} \times 0.511} \times 100 \% \\
Y_{G l u}=\frac{C_{G 0}-C_{G t}}{C_{G 0}} \times 100 \%
\end{gathered}
$$

where $Y_{E}$ is the actual ethanol yield, $C_{E t h}$ is the ethanol concentration in fermentation liquid, $C_{G 0}$ is the initial glucose concentration, and the 0.511 is the transformation coefficient of ethanol per gram glucose. $Y_{G l u}$ is the glucose utilizing ratio, and $C G_{0}$ is the initial glucose concentration, $C_{G t}$ is the final.

\section{RESULTS AND DISCUSSION}

\section{A. Ethanol Yield in Continuous Fermentation}

Ethanol yields in two reactors of two experimental groups were recorded to verify the influence of pre-activation and re-activation on ethanol production capacity of immobilized yeast cells in continuous fermentation. The ethanol yield of two reactors is presented in Fig. 1 and Fig. 2.

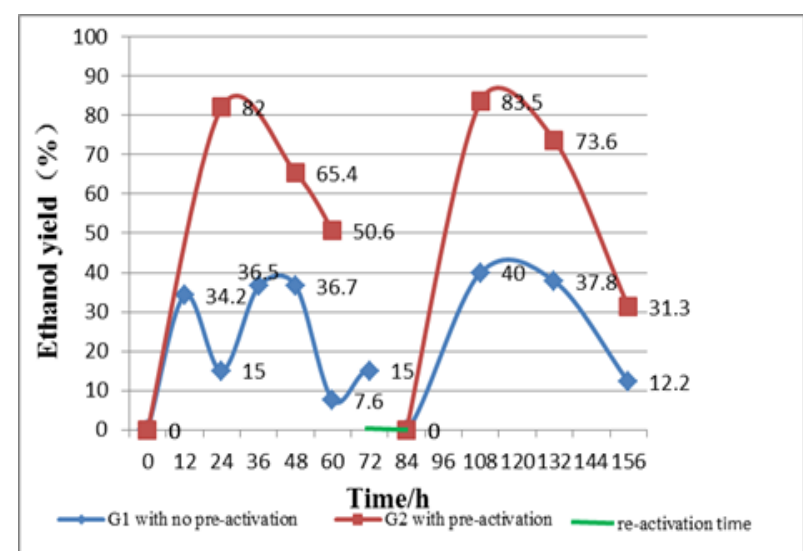

Fig. 1. Ethanol yield in 1\# reactor at fermentation time of $0-156 \mathrm{~h}$ with $12 \mathrm{~h}$ re-activation time and initial glucose concentration of $108.78 \mathrm{~g} / \mathrm{L}$.

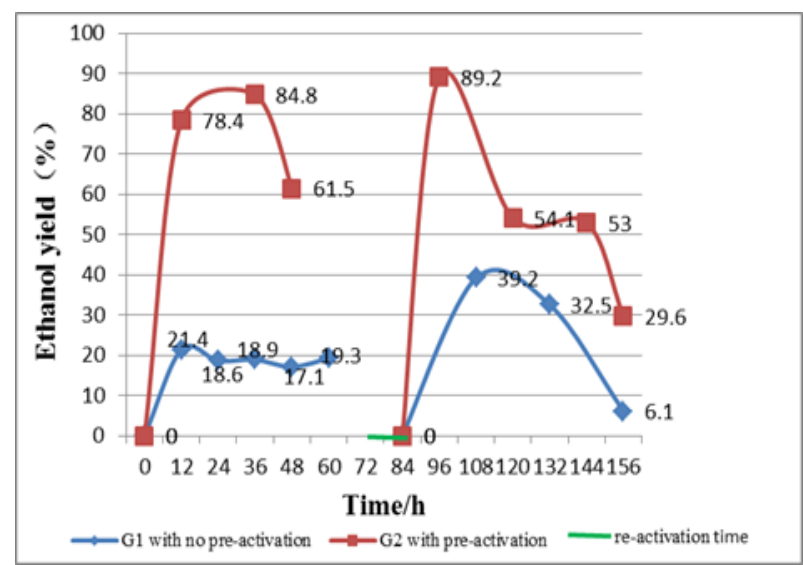

Fig. 2. Ethanol yield in 2\# reactor at fermentation time of $0-156 \mathrm{~h}$ with $12 \mathrm{~h}$ re-activation time and initial glucose concentration of $102.99 \mathrm{~g} / \mathrm{L}$.

Effect of pre-activation in continuous fermentation: The fermentation was performed in two reactors with same fermentation conditions in each experimental group. The trends of ethanol of two experimental groups were similar, with a sharp rise occurring within the first $36 \mathrm{~h}$ followed by a significant reduction. The yeast cells retained relatively high fermentation activation after being immobilized in calcium alginate beads, and immobilization didn't damage active center of cell. From the comparison of two experimental groups, the ethanol in G2 was apparently higher than that in G1. Pre-activation before fermentation enhanced cells' biological activity.

Effect of re-activation in continuous fermentation: The highest ethanol yield, $89.2 \%$, was obtained after re-activation time in $2 \#$ reactor of G2. The lowered ethanol yield obtained significant increase after reactivation. The trends of second period were similar to that in first period. After $72 \mathrm{~h}$ fermentation, the biological activity of cells reduced in reactors because of impurities, nutrition dead zones and other experiment reasons. Re-activation time of $12 \mathrm{~h}$ made the cell obtain original activity.

\section{B. Glucose Utilizing Ratio in Continuous Fermentation}

Glucose utilizing ratios of two reactors of experimental groups were studied to demonstrate biological activity changes of yeast cells. The glucose utilizing ratios of two reactors is presented in Fig. 3 and Fig. 4.

Effect of pre-activation in continuous fermentation: In each experimental group, the glucose utilizing ratios had a significant superiority in G2 with pre-activation than that in 
G1. The biological activity of immobilized yeast cells tended to be strengthened, and within first $36 \mathrm{~h}$, the ratio reached the highest, $95.93 \%$, which indicated that pre-activation cells could metabolize glucose effectively. With the increase of fermentation time, the glucose utilizing ratios of yeast had a significant decline, which in G1 fell from about $30 \%$ to $15 \%$ and in G2 fell from about $80 \%$ to $50 \%$. That indicated one-step activation might not maintain the high activity for long time because of variables in reactors.

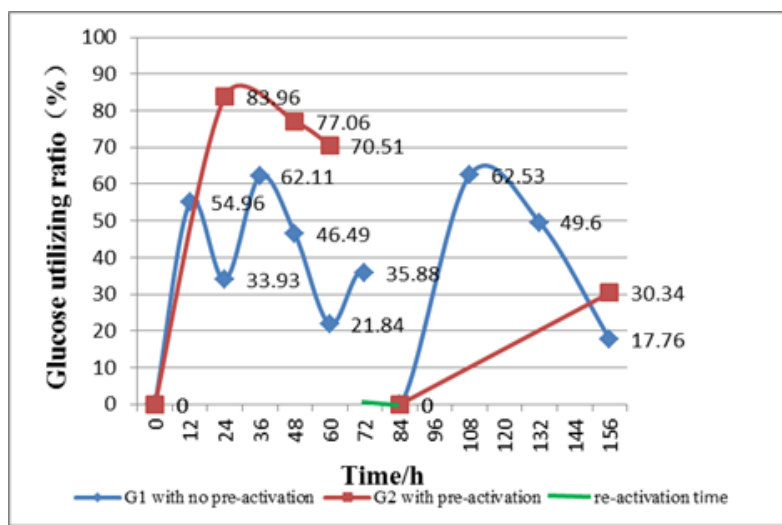

Fig. 3. Glucose utilizing ratio in $1 \#$ reactor at fermentation time of $0-156 \mathrm{~h}$ with $12 \mathrm{~h}$ re-activation time and initial glucose concentration in G1 was $109.78 \mathrm{~g} / \mathrm{L}$.

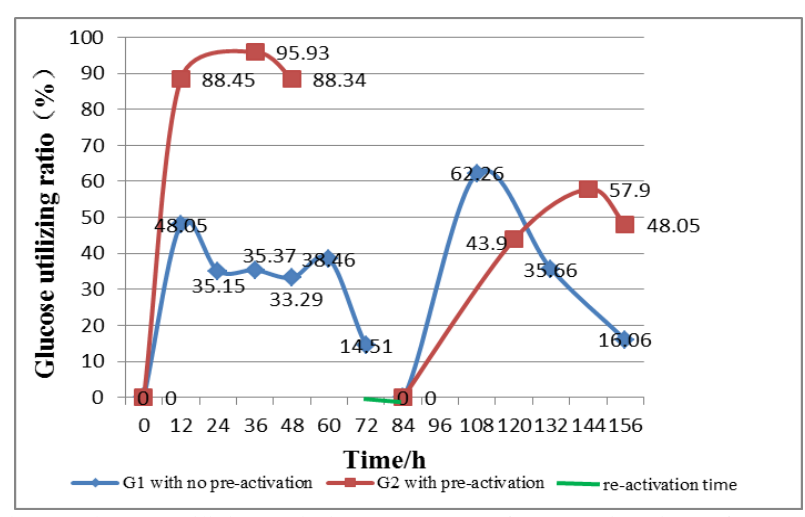

Fig. 4. Glucose utilizing ratio in 2\# reactor at fermentation time of $0-156 \mathrm{~h}$ with $12 \mathrm{~h}$ re-activation time and initial glucose concentration in G2 was $102.99 \mathrm{~g} / \mathrm{L}$.

Effect of re-activation in continuous fermentation: the glucose utilizing ratios of two reactors in each experimental group had a significant reduction after about $36 \mathrm{~h}$. After re-activation, the activity of immobilized yeast cells was regained with a rise trend of glucose utilizing ratio in the second period. At the end of each fermentation period, the glucose metabolism of yeast cells became inefficient, due to ethanol concentration in reactor, impurities after fermentation and mass transfer problems, which reduced the glucose utilizing ratio. After re-activation, yeast cells came alive due to the appropriate growth environment which stimulated the biological activity center.

\section{CONCLUSION}

In this study, an effective method for immobilization of the yeast $S$. cerevisiae BY4742 was applied in continuous fermentation. The ethanol yields and glucose utilizing ratios were analyzed in two groups of continuous fermentation reactors. Immobilized yeast had high ethanol fermentation activity but the performance was affected by many experiment factors, especially the biological activity of yeast [8]. Comparing date in G1 and G2, the ethanol yield could reach to about $80 \%$ in $\mathrm{G} 2$ with pre-activation; the glucose utilizing ratio had the similar pattern. Optimizing the growth condition showed satisfactory results [9], activation before the fermentation can significantly improve the efficiency of yeast producing ethanol; besides with the efficiency falling, yeast cells restore fermentation activity though re-activation. After re-activation, the glucose utilizing ratio could reach averagely $50 \%$. Ethanol yield within first $24-36 \mathrm{~h}$ could reach averagely $80 \%$ with pre-activation and re-activation. Activation enhanced the biological activity of cells thus improved the fermentation performance in continuous fermentation.

\section{REFERENCES}

[1] Y. Lin and S. Tanaka, "Ethanol fermentation from biomass resources current state and prospects," Applied microbiology and Biotechnology, vol. 69 , pp. 627-642, 2006

[2] S. Brethauer and C. E. Wyman, "Review: Continuous hydrolysis and fermentation for cellulosic ethanol production," Bioresource Technology, vol. 101, pp. 4862-4874, 2010.

[3] L. V. Reddy, L. P. Reddy, Y. J. Wee, and O. V. S. Reddy, "Production and characterization of wine with sugarcane piece immobilized yeast biocatalyst," Food and Bioprocess Technology, vol. 4, pp. 142-148, 2011.

[4] M. İnal and M. Yiğitoğlu, "Improvement of bioethanol productivity of immobilized Saccharomyces bayanus with using sodium alginate-graft-poly (N-vinyl-2-pyrrolidone) matrix," Applied Biochemistry and Biotechnology, vol. 168, pp. 266-278, 2012.

[5] A. Rattanapan, S. Limtong, and M. Phisalaphong, "Ethanol production by repeated batch and continuous fermentations of blackstrap molasses using immobilized yeast cells on thin-shell silk cocoons," Applied Energy, vol. 88, pp. 4400-4404, 2011.

[6] K. H. Lee, I. S. Choi, Y. G. Kim, D. J. Yang, and H. J. Bae, "Enhanced production of bioethanol and ultrastructural characteristics of reused Saccharomyces Cerevisiae immobilized calcium alginate beads," Bioresource Technology, vol. 102, pp. 8191-8198, 2011.

[7] Y. Lin, W. Zhang, C. Li, K. Sakakibara, S. Tanaka, and H. Kong, "Factors affecting ethanol fermentation using Saccharomyces Cerevisiae BY4742," Biomass and Bioenergy, vol. 47, pp. 395-401, 2012.

[8] C. Z. Liu, F. Wang, and F. Ou-Yang, "Ethanol fermentation in a magnetically fluidized bed reactor with immobilized Saccharomyces cerevisiae in magnetic particles," Bioresource Technology, vol. 100, pp. 878-882, 2009.

[9] B. Strel, S. Grba, and V. Maric, "Enhancement of biomass and fermentation activity of surplus brewers' yeast in a fed-batch process," Applied Microbiology and Biotechnology, vol. 39, pp. 53-57, 1993.

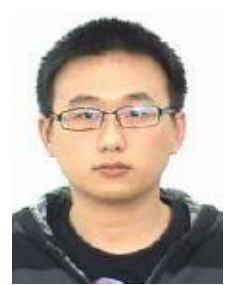

Zhaohui Xing is now a postgraduate student of Shanghai Jiao Tong University (SJTU), who was born in Nanjing, China on February 10, 1990. He obtained the bachelor degree in chemical engineering from Southeast University, China in 2013. His major research areas include ethanol fermentation from biomass wastes, biological treatment of wastewater.

He has been under the guidance of his mentor Prof. Lin since 2013 and his mainly research is in bio-resources and bioenergy.

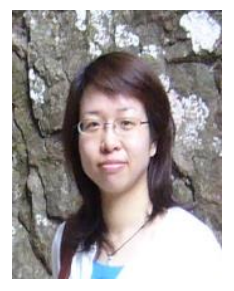

Yan Lin obtained her bachelor degree and master degree in environmental engineering from Xi'an University of Architecture and Technology, Shanxi, P.R. China in 1999 and 2002, respectively. Then she completed her PhD degree from Shanghai Jiao Tong University, Shanghai, P. R. China in 2005.

She was a postdoctoral fellow in Asian Center for Environment Research, Meisei University, Japan from 2005 to 2008. She worked in Shanghai Jiao Tong 
University since 2008, and she became an assistant professor. Her major research areas include ethanol fermentation from biomass wastes, biological treatment of wastewater.

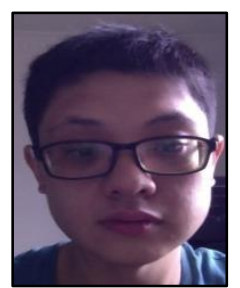

Qi Zhang is now a postgraduate student of Shanghai Jiao Tong University (SJTU), he was born in Shanghai China in 1998. He was majoring in environmental science and engineering and got his bachelor degree in SJTU in 2012.

He has been under the guidance of his mentor Prof. Lin since 2008 and his mainly research is in bio-resources and bioenergy. He has published two papers Chinese Social Sciences Citation Index (CSSCI) and one conference paper as the first author. Also, he has a patent application together with Prof. Lin (CHN 001310052631.3.).
Mr. Zhang performed well both in study and academic research so that he was recommended for a direct entry into postgraduate studies in SJTU without being required to sit any entrance examinations. He has been awarded the Tung OOCL Scholarship and the First Prize of SJTU academic scholarships in 2013.

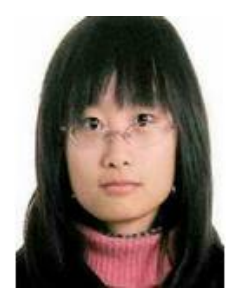

Xueyi Shi has studied in Shanghai Jiao Tong University for two years. She was born in Shanghai China in 1994. Her major field of study is environmental science and engineering.

She worked for Shanghai Environmental Monitoring Center as an Intern for 4 weeks. Also, she entered for a school project of providing tap water detection to neighbor housing estate. Her current research interest is water environment. 